\title{
Verweyen
}

Der Verkäufer der Zukunft -

Vom Drücker zum Beziehungsmanager und Teamplayer 
Alexander Verweyen

\section{Der Verkäufer der Zukunft - \\ Vom Drücker zum \\ Beziehungsmanager und Teamplayer}


Die Deutsche Bibliothek - CIP-Einheitsaufnahme

Verweyen, Alexander:

Der Verkäufer der Zukunft : vom Drücker zum

Beziehungsmanager und Teamplayer / Alexander

Verweyen. - Wiesbaden : Gabler, 1997

ISBN 978-3-322-91137-7

DOI 10.1007/978-3-322-91136-0

Der Gabler Verlag ist ein Unternehmen der Bertelsmann Fachinformation.

(C) Betriebswirtschaftlicher Verlag Dr. Th. Gabler GmbH, Wiesbaden 1997

Softcover reprint of the hardcover 1st edition 1997

Lektorat: Manuela Eckstein

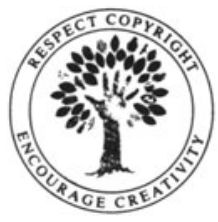

Das Werk einschließlich aller seiner Teile ist urheberrechtlich geschützt. Jede Verwertung außerhalb der engen Grenzen des Urheberrechtsgesetzes ist ohne Zustimmung des Verlages unzulässig und strafbar. Das gilt insbesondere für Vervielfältigungen, Übersetzungen, Mikroverfilmungen und die Einspeicherung und Verarbeitung in elektronischen Systemen.

http://www.gabler-online.de

Höchste inhaltliche und technische Qualität unserer Produkte ist unser Ziel. Bei der Produktion und Verbreitung unserer Bücher wollen wir die Umwelt schonen: Dieses Buch ist auf säurefreiem und chlorfrei gebleichtem Papier gedruckt. Die Einschweißfolie besteht aus Polyäthylen und damit aus organischen Grundstoffen, die weder bei der Herstellung noch bei der Verbrennung Schadstoffe freisetzen.

Die Wiedergabe von Gebrauchsnamen, Handelsnamen, Warenbezeichnungen usw. in diesem Werk berechtigt auch ohne besondere Kennzeichnung nicht zu der Annahme, daß solche Namen im Sinne der Warenzeichen- und Markenschutz-Gesetzgebung als frei zu betrachten wären und daher von jedermann benutzt werden dürften.

Umschlaggestaltung: Schrimpf und Partner, Wiesbaden

ISBN 978-3-322-91137-7 


\section{Vorwort}

Kundennähe, Kundenorientierung, Customer Focus und ähnlich lauten die Schlagworte, die die Unternehmensszene aktuell bestimmen. Ausgetüftelte Formen der Unternehmensorganisation wie Reengineering-Konzepte und Geschäftsprozeßoptimierung sollen dabei helfen, die entsprechenden Anforderungen umzusetzen. Doch regelmäßig bleiben die gewünschten Erfolge aus. Warum? Ganz einfach: Allzuoft wird bei den Programmen zur Steigerung der Kundennähe derjenige vergessen, der den Kunden am nächsten steht: der Verkäufer. Wenn sich der Verkäufer aber nicht ändert, bewirken auch die besten organisatorischen Konzepte wenig, denn der Verkäufer repräsentiert das Unternehmen, er ist der dirkete Ansprechpartner für die Kunden, er ist das Bindeglied zwischen Unternehmen und Markt.

\section{Kundennähe kann letzten Endes nur durch den Verkäufer im direkten Kontakt mit den Kunden realisiert werden.}

Stellen Sie sich einmal vor, Sie stecken eine immense Summe Geld in ein Organisationsentwicklungsprogramm, Ihr Marketing entspricht dem aktuellen State of the Art, aber Ihr Außendienst „funktioniert“ wie eh und je: „Einzelkämpfer“ machen sich auf den Weg, um durch standardisierte Vorgehensweisen den „König Kunde“ an seiner Monarchenwürde zweifeln zu lassen. Schade eigentlich um die hohen Investitionskosten. Etwas boshaft kann man sagen, daß die Verbesserung der Organisationsabläufe ohne die entsprechende Entwicklung der Verkaufsmannschaft dafür sorgt, daß der Kunde einfach schneller und effektiver verstimmt und vergrault wird.

Hiervor will Sie dieses Buch bewahren, denn in seinem Mittelpunkt steht der Verkäufer. 
Verkäufer sehen sich großen Herausforderungen und veränderten Anforderungen gegenüber. Gefragt ist deshalb der neue Verkäufer. Dieser plakative Begriff soll signalisieren, daß in der Aus- und Weiterbildung von Verkäufern endlich etwas passieren muß, daß neue Leistungsfaktoren und Verhaltensweisen vermittelt werden müssen so zählt zum Beispiel der klassische Einzelkämpfer bald schon zu den Auslaufmodellen, mehr und mehr ist der Teamworker gefragt. Doch dazu später mehr.

Gesucht wird also der neue Verkäufer - das Adjektiv „neu“ soll jedoch nicht signalisieren, daß Sie als altgedienter Verkaufsprofi sich einen neuen Job suchen müssen, um dem Verkäufer der Zukunft Platz zu machen. Im Gegenteil: Dieses Buch will Sie dazu auffordern und motivieren, Ihre Entwicklungspotentiale zu aktivieren, es möchte für anstehende Probleme sensibilisieren und praktikable Lösungen bieten. Der „neue“ Verkäufer ist auch der ,alte“ Verkäufer, der gelernt hat, sich den veränderten Aufgaben zu stellen und die Chancen, die der Wandel von Markt- und Unternehmenslandschaften bietet, zu ergreifen. 


\section{Inhaltsverzeichnis}

Vorwort.............................................. 5

Vom Drücker zum Beziehungsmanager

und Teamplayer...................................... 11

\section{Kapitel I: Herausforderungen}

für den Verkäufer der Zukunft......................17

Internationalisierung.................................... 19

Die Welt als Dorf ................................... 19

Die ,drei Nähen“" des Verkäufers........................ 20

Think Global .......................................... 21

Die Kultur des Kunden kennen......................... 22

Fragmentierung der Märkte.............................. 24

,Schnelle“ und komplexe Produkte ....................... 27

Im Mittelpunkt der Markt ................................ 28

Der Trend zur Dienstleistung........................ 28

König Kunde! ........................................ 28

König Kunde?.................................... 29

Die selbstverschuldete Servicewüste.................... 30

Es muß auch anders gehen ............................. 38

Unternehmensorganisation im Umbruch ................. 39

Es gibt viel zu tun .............................. 40

Kapitel II: Multitalent Verkäufer -

Die persönlichen Kompetenzen für den Erfolg......43

Ein exemplarisches Tätigkeitsprofil ....................... 45

Vier Verkäufertypen..................................... 47

Drei „Auslaufmodelle“ ... ...........................47

... und ein „Modell“" mit Zukunft........................49

Das richtige Selbstverständnis als Verkäufer............... 52

Den Abschluß im Fokus............................... 52

„Ja“ zur Verkäuferrolle sagen......................... 54

Rollenprobleme aufspüren ........................... 55

Jeder im Unternehmen ist Verkäufer................... 57

Das persönliche Leistungsprofil

der neuen Verkäufergeneration............................ 58

Abschlußstark durch Selbstbewußtsein................. 58 
Unternehmenskonformes Erscheinungsbild ............. 59

Höflichkeit: Eine Tugend am Hof von König Kunde..... 60

Die Sprache des Kunden sprechen.................... 62

Den Kunden überzeugen, nicht überreden ...............6 63

Mit positiver Ausstrahlung zu positiven Kundenkontakten 64

Durchsetzungsvermögen und Biß.................... 65

Partnerschaftlichkeit gleich Kundenfreundlichkeit ........66 66

Konfliktfähigkeit ................................ 70

Kompromißfähigkeit: Das Sieger-Sieger-Prinzip ........ 71

Teamgeist und Toleranz .............................. 72

Durchhaltevermögen: Der Verkäufer als Marathonläufer. . 73

Durch Engagement zum internen Unternehmer........... 74

Belastbarkeit: Knochenjob Verkaufen .................. 75

Mißerfolgstoleranz: Auf die Erfolge konzentrieren........ 77

Einzelkämpferfähigkeiten: Auch solo zum Erfolg......... 78

Entscheidungsfreude: Nicht zögern, sondern handeln.... 79

Dienstleistungsorientierung: Dem Kunden helfen wollen. 80

Wie sieht Ihr Leistungsprofil aus?..................... 82

Kapitel III: Neue Verkäufer haben System -

Visionen, Zeit und der Kunde ......................85

Gezielt zum Kunden und zum Erfolg ................... 87

Den Erfolg im Visier................................ 87

Visionen und Ziele formulieren........................ 88

Visionen als Kompaß............................. 92

Visionen motivieren................................ 93

Mehr Zeit für den Kunden:

Zeit- und Selbstmanagement ........................... 96

Zeit - eine wertvolle Ressource ....................... 96

Wo ist die Zeit geblieben?.............................. 98

Mehr aktive Verkaufszeit durch Tourenplanung.......... 109

Kundenorientierte Besuchsvorbereitung...................112

Die Analysephase: Der Verkäufer als Marktforscher......113

Die Zielsetzungsphase: Der Verkäufer

als Unternehmensberater ............................ 115

Die Strategiephase: Der Verkäufer als Kommunikator....116

Die Nachbereitungsphase: Der Verkäufer als Controller . 118 
Kapitel IV: Emotional Selling Kundenindividuelles Beziehungsmanagement .....119 Kundenkontakte mit Herz und Verstand...................121

Personal Selling und die verschiedenen Intelligenzen.....121

Haben Sie Ihre Gefühle im Griff?.......................124

Der Verkäufer mit Gefühl für sich selbst ... ............126

$\ldots$ und für seine Kunden ................................127

Vorsicht, Wahrnehmungsfalle!.......................130

Den Kunden motivieren....................................140

Primäre Motivation - der beste Weg

zur Begeisterung des Kunden..........................140

Die fünf Grundbedürfnisse Ihrer Kunden................142

So motivieren Sie die einzelnen ,Typen“ richtig...........148

Emotional Talk: Die nonverbale Kommunikation............159

Das nonverbale Dementi..............................159

Der „Kanal“ für Einstellungen und Gefühle...............160

Situationsmanagement ohne Worte....................162

Imagemanagement..................................163

Der „,konsistente“ Kunde .............................168

Kapitel V: Verkäufer im Team ....................171

Team-Selling ...........................................173

Einer für alle .......................................175

Gemeinsame Ziele, gemeinsame Werte,

gemeinsame Normen................................175

Von Angesicht zu Angesicht ..........................177

„We are the Champions" -

Die Phasen des Team-Building ........................178

Warum der Trend zum Team?............................180

Stabilität durch soziales Umfeld ..........................180

Leistungsplus ..................................... 180

Gebündelte Fähigkeiten...........................181

Probleme? - Nicht für gute Teams ......................182

Fit durch Feedback..................................183

Geborgenheit in der Gruppe ...........................183

Ich sehe was, das du nicht siehst....................183 
Die Tücken von Teamwork ............................. 185

Das Team als Insel ................................... 185

Team-Zwang...................................... 185

Marathon-Diskussionen ............................ 186

No risk, no fun .................................. 186

Talk im Team ...................................... 188

Kommunikationsnetze............................. 188

Wie offen kommuniziert das Team: Johari-Fenster ....... 189

Wer kann mit wem: Soziogramme ...................... 194

Der Weg zur Konfliktkultur............................ 197

\section{Kapitel VI: Das kundenorientierte Umfeld}

für Verkaufserfolge................................199

Der verkaufsaktive Innendienst..........................201

Verkäufer und Innendienst: An einem Strang ziehen .....201

Die Aufgaben des neuen Innendienstverkäufers.......... 202

Telefonmarketing ...................................210

Aktives Beschwerdemanagement ....................211

Die Verkaufskultur ..................................... 212

Bindemittel für dezentrale Strukturen..................212

Verkaufskultur als Chefsache.........................213

Mehrwert durch Kultur................................214

Schlußbemerkung ....................................215

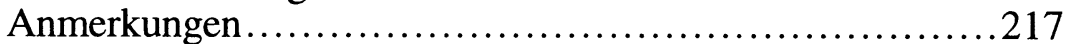

Literaturverzeichnis ...................................219

Der Autor....................................... 221 